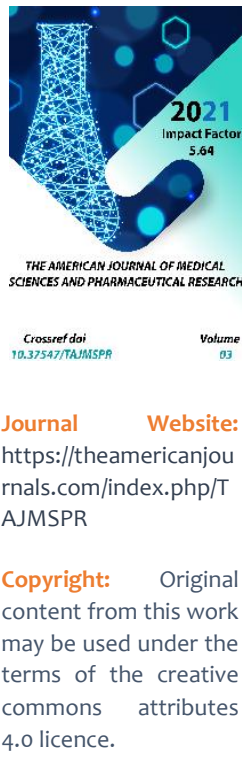

\title{
Effective Restoration Of Mandibular Defects With Custom- Made Titanium Implants
}

\author{
Feruz Ibodullaevich Tojiyev \\ Department Of Pediatric Oral And Maxillofacial Surgery, Tashkent State Dental Institute, \\ Tashkent, Uzbekistan
}

Aziz Mukhammadovich Azimov

Department Of Pediatric Oral And Maxillofacial Surgery, Tashkent State Dental Institute, Tashkent, Uzbekistan

\section{ABSTRACT}

Medical rehabilitation of patients with defects, deformities of the jaws is an urgent problem in reconstructive maxillofacial surgery. Such defects cause significant dysfunctions of the organs of the maxillofacial region, disfigurement of the soft tissues of the lower face zone. A complex problem of maxillofacial surgery is the development of methods for surgical restoration of the integrity of organs and their function. Currently, various surgical methods and materials are used to restore the defect in the jaw bone tissue [12].

\section{KEYWORDS}

Defects and deformities of the jaws, individually made titanium implants, autograft.

\section{INTRODUCTION}

Diagnosis and treatment of mandibular and maxillofacial soft tissue defects and deformities present a challenging problem. This is determined by the increasing number of injuries and oncological pathologies of the mandible, their combined nature, a significant number of unsatisfactory treatment results, low efficiency of traditional methods of surgical interventions using grafts and implants with deteriorating diagnosis. All of the above determines further search for ways to solve the problem of treatment planning, reconstruction and rehabilitation of patients with lower jaw defects and deformities $[5,6,7,8$,$] .$

For this purpose, autogenously, conserved allogeneic metatarsal and bone autografts, polymeric synthetic materials in the form of plastics, cements, and silicone compositions are used $[3,4]$. However, the experience of 
using each of the above-mentioned types of grafts and implants has revealed a number of drawbacks inherent in them. The analysis of the long-term results of the application of these plastic materials in the maxillofacial surgery revealed their disadvantages such as partial or complete resorption with the loss of the original shape and size, the suppuration of the operating wound with the rejection of the graft, displacement and encapsulation of the implants and others. Autologous grafts are susceptible to partial resorption with loss of their original shape and size, and their removal increases the trauma of surgical intervention $[7,11,14]$.

The planning of reconstructive surgery can be represented as a sequence of the following activities: identifying and analyzing the volumetric characteristics of the defect, determining the amount of lost tissue, deciding the choice of graft (autograft, allograft, implant, etc.), determining the donor site or source of other material, the method of transfer and fixation of the plastic material $[9,10]$.

When assessing the effectiveness of surgical treatment of patients with mandibular defects and deformities, special attention should be paid to the consideration of postoperative complications. Late postoperative complications are usually diagnosed after patients are discharged from hospital during the rehabilitation period. These patients are usually readmitted to the hospital or treated as outpatients. Most often these are purulent and inflammatory processes: suppuration of wounds, abscesses, fistulas. Rehabilitation of this group of patients requires a certain economic outlay. Late postoperative complications can occur days, months or even years later, if the body's resistance is reduced and the dormant infection is generalized $[2,13]$.

\section{MATERIALS AND METHODS}

To identify the causes of complications and to find out which method of surgical treatment is the most optimal, we conducted a comparative analysis of the treatment results of 44 patients with mandibular defects and deformities from 2016 to 2021 at the adult surgical dentistry and pediatric surgical dentistry of the Tashkent State Institute of Dentistry. The comparative assessment of treatment outcomes was based on clinical and radiological (orthopantomogram, MRI and MSCT) examinations and bed-day analysis. Analysis of the patient age distribution showed that the age group of 21 to 30 years old had the greatest number of patients (47.7\%). There is a slight predominance of women $-70.5 \%$ of the total number of patients. In 9 patients the defects occurred after oncological operations (various types of lower jaw resections). The number of patients with post-traumatic defects and deformities is 10, which indicates an increased number of injuries. These include the consequences of car accidents - 3, domestic and occupational injuries - 7, from secondary deforming osteoarthritis and ankylosis. In 19 patients, the defects and deformities occurred after the final stage of treatment of chronic osteomyelitis - sequestrectomy surgery, performed both in childhood and in adulthood. Severe and reflected anatomical and functional abnormalities of the maxillary system were observed during sequestrectomy surgery in childhood due to damage to the mandibular sprouts.

Congenital anomalies and deformities of the jaw comprised 6 people. Of the inferior 
micrognathia - 5, articular metaplasia - 1 patients.

Patients were divided into two groups: autotransplantation (rib, metatarsal bone) - 9 (20.5\%) patients and individual titanium implants - 35 (79.5\%) patients. The comparative assessment of the treatment results was based on the clinical and radiological examination and the analysis of the bed-days.

The clinical follow-up of all operated patients was followed up for 6 months to 1 year. The results were evaluated clinically, based on radiological findings, functional and aesthetic parameters and bed-day analysis.

While studying the 35 results of using custommade implants to replace the lower jaw defects, we observed a pronounced swelling of soft tissues in the post-operative period. In the postoperative period, all patients had marked swelling of the soft tissues in the area of surgical intervention, which lasted for a considerable period of time. In 9 cases, autografts (rib and metatarsal bone) were used to repair defects of the mandibular body, branch and articular process. Metatarsal bone (9 patients) was used to eliminate the defect of the branch and articular process of the lower jaw after ankylosis of the TMJ. In the case of significant mandibular defects, a rib was used. The number of patients in this case was 7 observations.

According to the data of X-ray check-up as well as functional and esthetic parameters in patients with rib autograft, in $25 \%$ (1 patient) we observed the rib partial resorption within 1, 5 to 5 years after the operation, which was caused by the discrepancy of bone architectonics of rib and lower jaw bones. Pyo-inflammatory complications were observed in 25\% (1 patient), and autograft displacement was 25\% (1 patient) When metatarsal bone was used to replace defects in the branch and articular process of the mandible, the number of autograft displacements was $16.67 \%$ (1 observation). Inflammatory complications were observed in $16.67 \%$ (1 patient). Inflammatory complications were detected in more than half of the cases, all patients had severe pain and swelling in the postoperative period at the site of the autograft, which increased the length of stay in hospital. The average length of stay in hospital for patients in this group was 9.3 days. They were treated as outpatients for 58 days due to additional trauma to the patient. To prevent the development of complications, we have developed a working scheme, the main principle of which is to take into account errors at each stage of treatment of a patient with mandibular defects and deformities. The approach is conventionally divided into tactical and technical approaches. In the preoperative period, the following approaches can be made: insufficient preparation of the patient for surgery (un eliminated foci of chronic infection in the body, no prevention of exacerbations and treatment of concomitant diseases), the approach to implant manufacturing (the size of the defect is not taken into account, improper manufacturing, which changes the physical and chemical properties of the material), choosing the wrong treatment plan for the patient, wrong choice of analgesia. In the intraoperative period, these include poor surgical technique, improperly shaped implant bed, leaky isolation of the implant from the mouth, foreign bodies left behind, traumatic handling of the defect area, inadequate or excessive surgical scope, and anaesthetic management. And in the postoperative period, respectively, poor wound care and inadequate oral hygiene, inappropriate choice of antibiotic 
therapy (wrong dosage and timing, combination of drugs, no consideration of sensitivity, etc.), no repeated laboratory tests after surgery or no consideration of their results, inadequate infusion and general restorative therapy. Based on the above, it is evident that the selection of the optimal method of treatment for this pathology should be based on the results of a thorough preoperative examination of the patient.

In order to identify and analyze the volumetric characteristics of the bone skeletal defect, most oral and facial surgeons still use only methods based on photographs of the patient in several projections, plaster masks and X-rays of the mandibular bones in standard stacks in everyday practice. However, the data obtained from these methods are tentative, which leads to errors in surgical planning and the occurrence of complications [1]. According to foreign literature, the best imaging method currently available is the multispiral computed tomography technique. The use of multispiral computed tomography with $3 \mathrm{D}$ reconstruction allows the most complete representation of the boundaries, size and volume of the defect or deformity, the precise localization and degree of displacement of bone fragments, and the condition of the bony structures of the TMJ [5].

To confirm the effectiveness of MSCT examination in patients with mandibular defects and deformities, 42 patients were examined on an Aquilion 128 multispiral CT scanner by TOSHIBA in axial, coronal and frontal projections, who were scheduled for complex reconstructive and restorative operations on the lower jaw bones. As a result of the additional information obtained, a treatment tactic was determined for each individual case. The studies helped to determine the optimal method of surgical intervention, which, in turn, made it possible to reduce the time of surgical intervention and improve the level of anatomical and functional rehabilitation of the patients. Application of the proposed improvement in the protocol of patients' examination and in the technique of reconstructive operations allows optimizing treatment, avoiding a large number of complications and reducing the period of treatment and, especially, the examination of the patient.

\section{RESULTS OF THE STUDY}

Thus, the three-dimensional computer models of the lower jaw bones obtained as a result of the studies allowed us to accurately assess the boundaries of the defects, the degree of bone displacement and localization, and the degree of bone structure deformation.

Summarizing the above, we can conclude that multispiral computed tomography is a universal diagnostic apparatus of the new generation with great potential for use in maxillofacial surgery. The application of CAD, $R$ \& $D$ methods in the medical field, is an invaluable contribution of Mechanical Engineering Technology to medicine. The ability to produce physical models directly from scanned data, promises to be the image of the future in medical surgery. In the coming years, parts/inserts are expected to be 'created' to complement non-functional parts of the human body. Advances in RP material undoubtedly allow the production of 'clean' parts that can be used directly as implants. It is the possibility of such technical methods of production of complex 'designs' combined with advances during surgical interventions that will allow replacement of human body parts, reconstruction of others, and carrying 
out operations with great precision. In the process of individual design of implants for each patient, based on $\mathrm{CT}$ data has been proven to be feasible and promising. However, further research is needed to reduce the cost of such a process. If the process of individual design and manufacture of implants based on specific patient data can be further simplified and the cost is reduced to an acceptable level, this could be a common process for the future. With the ability to design and produce any type of implant at low cost, no more mass production from implants would be necessary. By combining specially designed implants with newly developed orthopaedic surgical robots, the advantages can be further extended.

\section{CONCLUSIONS}

The obtained results of four-year follow-up, controlling the level of anatomical and functional rehabilitation, show that a custommade implant made of titanium allows to avoid complications, arising during implant fitting, due to the possibility of height and position adjustment. Besides, in comparison with autografts, the patient doesn't suffer from additional trauma, thus, the surgical aggression and risk of complications development is decreased.

\section{REFERENCES}

1. Kazakov, S.V. (2004). Orthopedic treatment of patients with jaw defects. Experimental clinical research (Doctoral dissertation, Perm State Medical Academy). Perm, 159 p.

2. Kozlov, V. A., \& Semenov, M. G. (2003). Bone-reconstructive treatment of congenital and priobretenny deformations of people in childhood.
Uchebnoe posobie. Publishing house SPb MALO.-SPb. 36 p.

3. Mamedov, E.V., \& Verbo, E.V. (2003). Use of free vascularized autografts from the lateral chest wall in maxillofacial surgery. Dentistry, (1), 5254 .

4. Nigmatullin, R.T. (2003). Essays on Tissue Transplantation: A Course of Lectures for Physicians. Ufa: Polygraph plant. p.160.

5. D.Sh. Devdariani, A.B. Alexandrov, A.V. Kulikov, I. V. Baranov. (2005). Features of bone grafting of the lower jaw by intraoral access. $X$ International Conference of Maxillofacial Surgeons and Dentists: Conference Proceedings. S-Pb. P.48.

6. Semenov, M.G. (2005). Bone reconstructive treatment of tissue developmental abnormalities and acquired deformities of the maxillofacial area in children (Doctoral dissertation, State educational institution of additional professional education "St. Petersburg Medical Academy of Postgraduate Education of the Ministry of Health of Russia"). 36 p.

7. Shomurodov K.E., Isomov M.M. (2020). Monitoring of inpatient and outpatient rehabilitation of pregnant women with inflammatory diseases of the PMO. Tashkent. Dentistry, No. 1, pp. 34-37.

8. Farwell, D. G., \& Futran, N. D. (2000). Oromandibular reconstruction. Facial plastic surgery, 16(02), 115-126.

9. Hurmerinta, K., \& Hukki, J. (2001). Vector control in lower jaw distraction osteogenesis using an extra-oral multidirectional device. Journal of Cranio-Maxillofacial Surgery, 29(5), 263-270. 
10. Saeed, N. R., Cunliffe, D. R., \& WattSmith, S. R. (2002). Role for sulcus deepening sutures in reconstructive surgery. The British journal of oral \& maxillofacial surgery, 40(3), 262.

11. Shokirov Sh.T. (2020). The technique of bimaxillary osteotomy with the help of intermediate kappa-splints for the elimination of upper retrognathia in patients with congenital clefts of the upper lip and palate. The American Journal of Medical Sciences and Pharmaceutical Research. pp.81-93.

12. Shomurodov. K.E. (2020). Features of cytokine balance in gingival fluid at odontogenicphlegmon of maxillofacial area. Doctor-aspirant. 42(5.1). P.187-

192.

13. Shomurodov, K. E. (2020). Comparative evaluation the anatomical and functional state of the. Journal of research in health science, 1(4), 54-57.

14. Djuraeva, D. D., \& Berdiyeva, Z. M. (2016). Cultural heritage as a factor of human development (on the example of Uzbekistan). Ученый XXI века, 23. 